\title{
Apport Du SIG Et De L'analyse Multicritère Dans La Prospection Hydrogéologique Du Socle Précambrien De Gagnoa (Centre-Ouest De La Côte d'Ivoire)
}

\author{
Onétié Zahibo Oscar, MA \\ Lasm Théophile, PhD \\ Coulibaly Adama, PhD \\ Baka Derving, PhD
}

Laboratoire des Sciences et Techniques de l'Eau et de l'Environnement, UFR STRM, Université Felix Houphouët-Boigny, Côte d'Ivoire

Fossou N'guessan Marie Rosine, PhD

Laboratoire Géosciences et Environnement, UFR SGE, Université Nangui Abrogoua, Côte d'Ivoire

\section{Youan Ta Marc, PhD}

Laboratoire des Sciences et Techniques de l'Eau et de l'Environnement, UFR STRM, Université Felix Houphouët-Boigny, Côte d'Ivoire Centre Universitaire de Recherche et d’Application en Télédétection

(CURAT), Université Felix Houphouët-Boigny, Côte d'Ivoire

\section{Yao Kouadio Assemien François, MA}

Laboratoire des Sciences et Techniques de l'Eau et de l'Environnement,

UFR STRM, Université Felix Houphouët-Boigny, Côte d'Ivoire.

Ecole des Mines d'Alès, Alès (France)

Aoulou Kouassi Aristide, MA

Laboratoire des Sciences et Techniques de l'Eau et de l'Environnement,

UFR STRM, Université Felix Houphouët-Boigny, Côte d'Ivoire

doi: 10.19044/esj.2016.v12n23p137 URL:http://dx.doi.org/10.19044/esj.2016.v12n23p137

\begin{abstract}
Côte d'Ivoire is constituted in more than 97.5\% of crystalline basement which contains the main part of groundwater resources of the country. These resources are contained in the weathering material and fissured aquifers. Gagnoa area located at the Central West of Côte d'Ivoire, on crystalline and metamorphic basement. In this area, surface water and groundwater are exploited for the water supply of the populations. However surface water are often doubtful quality and can affect the health of these
\end{abstract}


populations. Thus find water resources in sufficient and good quality amount is a necessity in this area. The study aims to identify favourable areas for the archievement of drillings with important discharge using geomorphologic, hydrogeological data and remote sensing. Multi-criteria analisys methods were used in this study. The database is constituted by various data in particular, data sheets of drillings, hydroclimatic statements and satellite image. This database was stored, analyzed, treated and managed using ARCGIS 10.2. software.

Various treatments allowed to establish various thematic maps (accessibility, exploitability, availability). The synthesis of all information inside a SIG allowed to establish the maps of favourable areas to achieve drillings with large discharges. The classes of good and excellent sensibility cover $26 \%$ of the study area. These favourable areas for the achievement of drillings with important discharge are located at the center and south of the study area. Validation of this map showed that $66 \%$ of the water points (drillings) with discharge superior to $5 \mathrm{~m}^{3} / \mathrm{h}$ overlap on the good and excellent classes of sensibility. The various zones of productivity of Gagnoa area were able to be characterized. These results are very interesting and useful for the hydrogeological prospecting. They contribute to a better knowledge and a groundwater resource management.

Keywords: Groundwater ressources, Multi-criteria analysis, SIG, hydrogeological prospecting, Gagnoa

\section{Résumé}

La Côte d'Ivoire est constituée à plus de 97,5\% de socle qui renferme l'essentiel des ressources en eaux souterraines du pays. Ces ressources sont contenues dans les aquifères fissurés et des altérites. La région de Gagnoa est située au Centre-Ouest du pays sur un substratum cristallin et cristallophyllien. Dans cette région, les eaux de surface et souterraines sont exploitées pour l'approvisionnement des populations. Cependant les eaux de surface sont souvent de qualité douteuse et peuvent affecter la santé des populations. Trouver des points d'eau en quantité suffisante et de bonne qualité est donc une necessité dans cette région. La présente étude a pour objectif d'identifier des zones favorables à l'implantation de forages à gros débits en couplant les données géomorphologiques, hydrogéologiques et de télédétection. La base de données est constituée de fiches techniques de forages, des relevés hydroclimatiques et des images satellitaires. La méthode de l'analyse multicritère a été utilisée dans cette étude. Cette base de données a été stockée, analysée, manipulée et gérée par le logiciel ARCGIS 10.2. Les différents traitements ont permis d’établir différentes cartes thématiques (accessibilité, exploitabilité, disponibilité). La synthèse de toutes ces 
informations à l'intérieur d'un SIG a permis d'établir la carte des zones favorables à l'implantation de forages à gros débits. Les classes de sensibilité bonne et excellente couvrent 26\% de la zone d’étude. Ces zones favorables à l’implantation de forages à gros débits sont localisées au Centre et au Sud de la région étudiée. La validation de cette carte a montré que $66 \%$ des points d'eau (forages) de débits supérieurs à $5 \mathrm{~m}^{3} / \mathrm{h}$, se superposent aux classes de sensibilité bonne et excellente. Les différentes zones de productivité de la région de Gagnoa ont pu être caractérisées. Ces resultats sont fort intéressants et utiles pour la prospection hydrogéologique. Ils contribuent à une meilleure connaissance et gestion des ressources en eaux souterraines.

Mots clés : Ressources en eaux souterraines, analyse multicritère, SIG, prospection hydrogéologique, Gagnoa

\section{Introduction}

L’approvisionnement des populations en eau potable en qualité et en quantité constitue un véritable challenge pour les pays en développement comme la Côte d'Ivoire. La région de Gagnoa qui fait l'objet de cette étude a bénéficié de nombreux programmes d'hydrauliques dont la construction d'un barrage pour desservir les populations en eau potable. Cependant, cette retenue d'eau est non seulement exposée à de nombreuses sources de pollution (les déchets, effluents domestiques, les engrais utilisés pour les cultures maraichères et au phénomène d'eutrophisation) (Eba, 2013) mais n’arrive pas à couvrir les besoins en eau des populations surtout rurales. En effet, la région a connu un taux d’accroissement élevé (13\%) accentué par la crise socio-politique déclenchée en 2002. Un recensement de la Société de Distribution d'Eau de Côte d'Ivoire (SODECI) réalisé dans la région en 2002 montre que le taux de couverture était de 50\%. Les populations rurales ont recours aux eaux de puits et des rivières pour satisfaire leurs besoins en eau, malgré leur qualité souvent douteuse (30\% des ouvrages d'hydrauliques sont des puits à grand diamètre) et leur état bactériologique désastreux (Lasm, 2000). L'utilisation des eaux souterraines représente par conséquent une alternative pour l'alimentation en eau potable dans la région. Cette ressource présente l'avantage d'être à l'abri des fluctuations saisonnières et d'éventuelles sources de pollution (Lasm, 2000). Les eaux souterraines constituent l'essentiel des ressources en eau de notre pays dont le socle occupe $97,5 \%$ du territoire. En milieu cristallin et cristallophyllien, les eaux se trouvent dans deux réservoirs principaux: d'une part les réservoirs des altérites ayant une fonction essentiellement capacitive et d'autre part les réservoirs de fissures et de failles plus profonds ayant une fonction conductrice (Wyns et al., 1999). Le taux d’échec enregistré au cours des projets hydrauliques dans la région est en moyenne de $30 \%$ avec un pic 50\% 
pour le projet BID-2 (2008-2010). Les ouvrages hydrauliques déjà réalisés ont des débits en général faibles ( $80 \%$ des forages) et sont inégalement répartis. Le fort taux d'échec dans le cadre de ces projets est dû en plus de la méconnaissance des milieux fractures, à un manque d'études poussées lors des prospections. Des études réalisées par Langevin et al. (1991) en France, Nakolendousse et al. (1993) au Burkina Faso ainsi qu'en Côte d'Ivoire par Saley (2003), Jourda (2005), Youan Ta (2008), Youan Ta et al. (2011, 2015), Akossi et al. (2014) ont proposé une approche de prospections hydrogéologiques. Cette approche permet de coupler d'une part la géologie, la géomorphologie, l'hydrogéologie et la télédétection (photographie aérienne, imagerie satellitaire, radars) et d'autre part le SIG et la méthode d'analyse multicritère. La combinaison de ces méthodes a permis de cartographier des zones propices à l'implantation de forages à gros débits dans diverses régions de la Côte d'Ivoire et notamment dans les régions semi-montagneuse de Man (Saley, 2003), de Daoukro (Kanohin et al., 2012), des Savanes (Jourda, 2005), de Bondoukou (Youan Ta, 2008; Youan Ta et al., 2011) et d'Agnéby (Dibi, 2008). C'est dans cette perspective que s'inscrit ce travail qui a pour but d'identifier des zones favorables à l'implantation de forages à gros débits dans la région de Gagnoa avec l'apport du SIG et de l'analyse multicritère. Elle intéresse une zone de socle de la Côte d'Ivoire où la fracturation est développée et les études hydrogéologiques sont très fragmentaires.

\section{Présentation de la zone d'étude}

Situé au Centre-Ouest de la Côte d'Ivoire, entre $5^{\circ} 40$ et $6^{\circ} 10$ de latitude Nord et entre $5^{\circ} 50$ et $6^{\circ} 20$ de longitude Ouest, le département de Gagnoa est le chef-lieu de la région de Gôh. Elle couvre une superficie de $2500 \mathrm{~km}^{2}$ avec une population estimée à 897117 (INS, 2014). La géomorphologie est marquée par une pénéplaine ondulée. La végétation dominante est la forêt dense. La région est située en zone humide avec une pluviométrie moyenne annuelle de $1300 \mathrm{~mm}$. Elle est caractérisée par quatre saisons, deux saisons des pluies et deux saisons sèches. Le réseau hydrographique est dense avec pour cours d'eau principal le Guéri. Le substratum de la région de Gagnoa est composé de roches cristallines et cristallophylliennes constituées principalement de granitoïdes et de formations volcano-sédimentaires. Les formations volcano-sédimentaires sont représentées par des amphibolites à grains fins et à structure nettement schisteuse, des schistes chloriteux, sériciteux et des tufs de couleur gris-vert avec des grains généralement très fins. Les granitoïdes les plus importants sont les granites et les granodiorites orientés et non orientés. Ces différentes formations géologiques ont été structurées au cours de l'orogenèse éburnéenne et sont d’âge protérozoïque. Selon la carte de Tagini (1972), elle 
ne présente pas assez de données structurales, sauf quelques failles trouvées orientées NO-SE et localisées pour la plupart dans le Nord-Ouest de la région. Au plan hydrogéologique, l'on peut distinguer trois types de réservoirs : des réservoirs d'altérites ; de fissures et de fractures majeures.

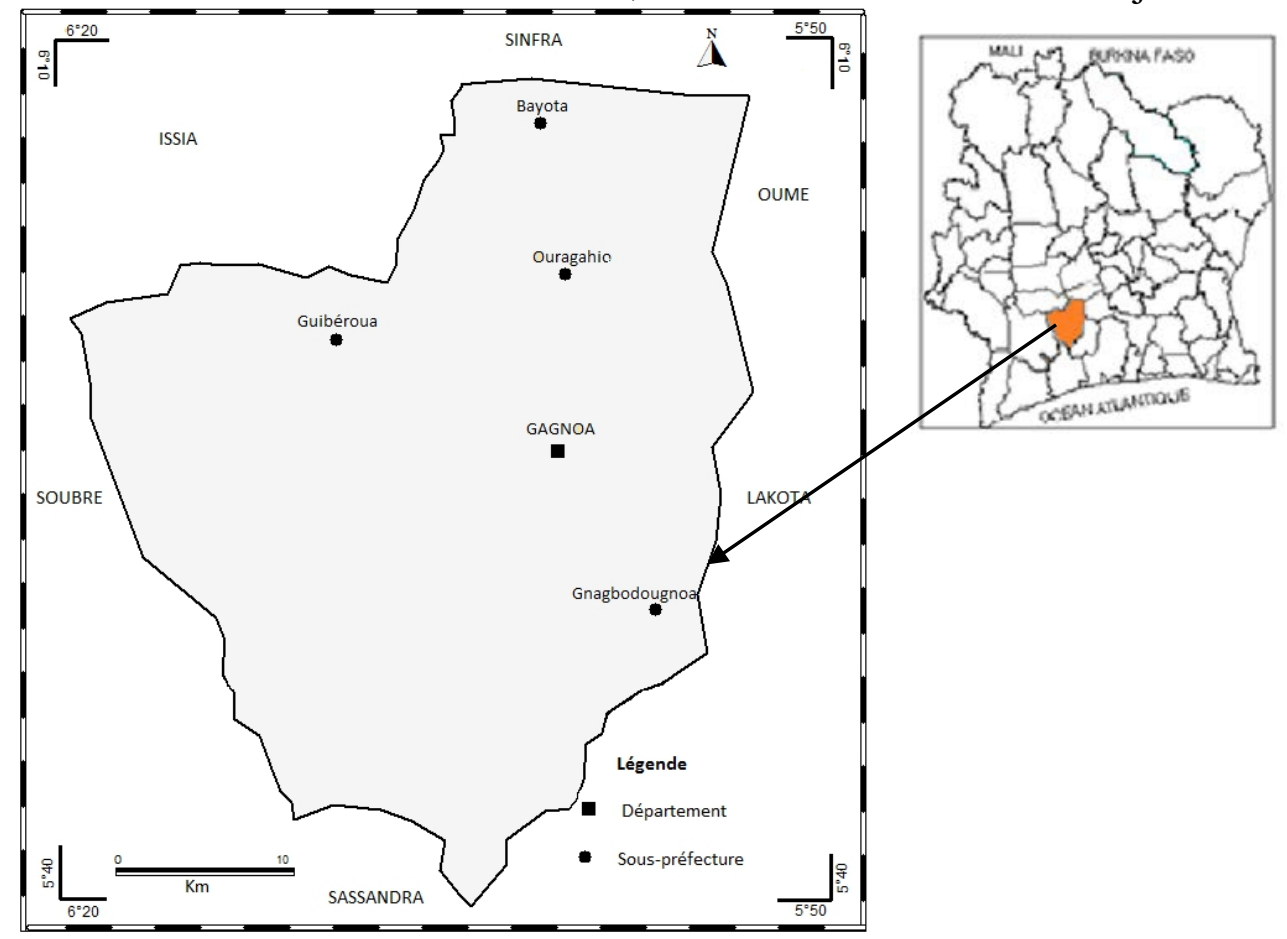

Figure 1: Présentation de la zone d'étude

\section{Matériel et Méthodes \\ Données}

Cette étude a nécessité l’utilisation de plusieurs types de données dont les données climatiques constituées de précipitations mensuelles et annuelles (1966-2008) recueillies à la station météorologique de Gagnoa et fournies par la SODEXAM; de cartes topographiques au 1/200 000 des degrés carrés de Gagnoa, Daloa, Grand-Lahou et Soubré éditées par l’Institut de Géographie National France (IGN) (1968), d'images satellitaires ETM+ de Landsat-7 (197-55 et 197-56) qui ont été utilisées pour la cartographie du réseau linéamentaire et 154 fiches techniques de forages établies lors du récent programme d’hydraulique villageoise BID (2008-2010).

\section{Méthodologie}

Dans le cadre de cette étude, la méthode d'extraction des linéaments par traitement des images satellitaires ne sera pas abordée car très connue, le lecteur pourra consulter l'abondante littérature disponible à cet effet (Jourda et al., 2006 ; Youan Ta et al., 2008; Sorokoby et al., 2010). Ici il s’agira de 
mettre l'accent sur la méthode de cartographie thématique des ressources en eau. Cette méthodologie comporte différentes étapes :

-l'identification des critères de décisions, la classification et la standardisation de ces critères en vue de l'élaboration des indicateurs conformément aux objectifs à atteindre ;

-la pondération des critères et leur agrégation suivant la démarche multicritère.

\section{Identification des critères de l'analyse multicritère}

En se référant aux observations de terrain et aux travaux d’El Morjani (2002), Hentati et al. (2005), Jourda (2005), Kouamé (2007), Youan Ta (2008), un certain nombre de critères a été identifié, sélectionné et évalué pour l'établissement des différentes cartes thématiques conformément aux objectifs fixés ; à savoir l'établissement des cartes thématiques concernant les indicateurs de disponibilité en eaux souterraines, d'accessibilité, d’exploitabilité. Pour cette étude, les critères jugés importants sont la densité de drainage ( $\mathrm{km} / \mathrm{maille})$, la densité de fracturation ( $\mathrm{km} / \mathrm{maille})$, l'altitude (m), l'épaisseur d'altération (m), l'infiltration efficace $(\mathrm{mm})$, le coût de foration (F CFA), la probabilité de réussite (\%), le débit d'exploitation $\left(\mathrm{m}^{3} / \mathrm{h}\right)$ et le niveau statique $(\mathrm{m})$.

\section{Classification et standardisation des critères}

La classification des critères doit tenir compte de la variance des données et s'inspirer de la classification adoptée lors des travaux antérieurs (Jourda, 2005 ; Jourda et al., 2006) réalisés en région de socle et de la classification proposée par le C.I.E.H (Comité Inter-Africain d’Études Hydrauliques) pour les débits. Pour une meilleure interprétation le nombre de classes a été réduit à 5 à savoir : très faible ; faible; moyenne ; forte ; très forte. Une standardisation des critères est obligatoire car les différents critères sont mesurés en diverses unités et selon différentes échelles.Un intervalle commun de 0 à 10 (Jourda, 2005, Jourda et al., 2006 ; Kouamé, 2007) a été retenu. La note 10 est attribuée aux classes « très faible » ou " très forte » selon qu'elles contribuent à l'excellente réalisation de l'indicateur considéré. Dans le cas inverse, la note 1 est attribuée à ces classes. Des valeurs intermédiaires sont attribuées aux autres classes selon une distribution linéaire. Le tableau I présente la classification des critères pour la réalisation de la carte thématique accessibilité. 
Tableau I : Classification et standardisation des critères d'accessibilité des ressources en eaux souterraines.

\begin{tabular}{|c|c|c|c|c|}
\hline Indicateur & critères & $\begin{array}{l}\text { Qualificatifs des } \\
\text { critères }\end{array}$ & Classes & Note \\
\hline \multirow{10}{*}{ Accessibilité } & \multirow{3}{*}{ Probabilité } & Trèsfaible & $<24$ & 1 \\
\hline & & Faible & 24- 41 & 3 \\
\hline & & Moven & $41-57$ & 5 \\
\hline & \multirow{2}{*}{ de succès } & Fort & $57-74$ & 8 \\
\hline & & Très fort & $>74$ & 10 \\
\hline & \multirow{3}{*}{ Coût de } & Trèsfaible & $<1188850$ & 10 \\
\hline & & Faible & 1188850-1379950 & 8 \\
\hline & & Moven & $1379950-1571700$ & 5 \\
\hline & \multirow{2}{*}{ foration } & Elevé & $1571700-1763450$ & 3 \\
\hline & & Très élevé & $>1763450$ & 1 \\
\hline
\end{tabular}

\section{Pondération des critères}

La pondération des critères de décision a été effectuée suivant la méthode de comparaisons par paire par le processus d'analyse hiérarchique (AnalyticalHierarchyProcess, AHP) développée par Saaty (2000) et utilisée par Dibi (2008), Youan Ta et al. (2011). Les coefficients de pondération des facteurs sont déterminés à partir d'une série de comparaisons par paire en tenant compte de leur importance déterminée sur une échelle numérique de 9 niveaux proposée par El Morjani (2002). En utilisant la matrice générée par la comparaison par paire sur les valeurs, des vecteurs propres sont obtenues en calculant leur moyenne géométrique par ligne selon l'équation 1 :

$$
v p=\sqrt[n]{\prod_{i}^{n} g i}
$$

avec

vp : vecteur propre ;

$\mathrm{n}$ : nombre de critère ;

gi : note du critère i.

Le coefficient de pondération pour chaque critère est obtenu en divisant chaque vecteur propre par leur somme. Des exemples de calculs sont insérés dans le tableau II.

Tableau II : Matrice de comparaison par paire et coefficient de pondération des critères de l'indicateur accessibilité

\begin{tabular}{ccccc}
\cline { 2 - 5 } & $\begin{array}{c}\text { Probabilité de } \\
\text { succès }\end{array}$ & $\begin{array}{c}\text { Coût de } \\
\text { foration }\end{array}$ & Vecteur propre & $\begin{array}{c}\text { Coefficient de } \\
\text { pondération }\end{array}$ \\
\hline $\begin{array}{c}\text { Probabilité de } \\
\text { succès }\end{array}$ & 1 & $1 / 3$ & $\sqrt{1 * 1 / 3}=0,58$ & $\frac{0,58}{1,73+0,58}=0,25$ \\
\hline $\begin{array}{c}\text { Coût de } \\
\text { foration }\end{array}$ & 3 & 1 & $\sqrt{3 * 1}=1,73$ & $\frac{1,73}{1,73+0,58}=0,75$ \\
\hline
\end{tabular}


Une fois la matrice définie, il faut vérifier la cohérence du jugement porté par le ratio de cohérence (RC). Le ratio de cohérence se calcule par le rapport de l'indice de cohérence (IC) et l'Indice Aléatoire (IA) proposé par Saaty (1977). Un raisonnement est dit cohérent si son ratio de cohérence est inférieur ou égal à 10\%. Les valeurs de l'indice aléatoire (IA) sont consignées dans le tableau III. Ces valeurs sont données en fonction du nombre de paramètres comparés.

Tableau III : Indices aléatoires en fonction du nombre d'éléments comparés (Saaty, 1977)

\begin{tabular}{lccccccccc}
$\begin{array}{c}\text { Nombre d'éléments } \\
\text { comparés }\end{array}$ & 2 & 3 & 4 & 5 & 6 & 7 & 8 & 9 & 10 \\
\hline Indice aléatoire (IA) & 0 & 0,58 & 0,90 & 1,12 & 1,24 & 1,32 & 1,41 & 1,45 & 1,49 \\
\hline
\end{tabular}

La détermination de l'indice de cohérence d'une matrice de jugement se résume en ces étapes :

- $\quad$ multiplier chaque colonne de la matrice par le poids ou le coefficient de pondération lui correspondant ;

- $\quad$ sommer les éléments de chaque ligne de la matrice ;

- $\quad$ diviser chaque total de ligne par le poids du critère correspondant à cette ligne ;

- déterminer $\lambda$ max qui est la moyenne des résultats obtenus à l'étape précédente ;

- $\quad$ déterminer l'indice de cohérence avec la formule suivante :

IC $=\frac{\lambda \max -\text { nombre de colonne }}{\text { nombre de colonne-1 }}$

Dans cette étude, le ratio de cohérence (RC) de la matrice de comparaison par paire de l'indicateur disponibilité est de 2,89\%. Le ratio RC est inférieur à $10 \%$ donc la matrice est cohérente par conséquent les coefficients de pondération obtenus peuvent être utilisés comme poids pour l'établissement de l'indicateur disponibilité.

\section{Agrégation des critères : analyses multicritères}

Dans cette étude, la méthode d'agrégation par pondération a été utilisée. Celle-ci consiste à sommer les valeurs standardisées et pondérées de chaque critère intervenant dans l'élaboration d'un indicateur donné (Youan Ta, 2008 ; Youan Ta et al., 2011). L'équation [3] illustre bien ce propos:

$$
\mathrm{S}=\sum_{\mathrm{i}=2}^{\mathrm{n}} \mathrm{W}_{\mathrm{i}} \mathrm{X}_{\mathrm{i}}
$$

avec :

$\mathrm{S}$ : somme ;

Wi: poids du critère i ;

Xi: valeur standardisée du critère de facteur i. 
L'établissement de la carte d'un indicateur donné consistera à reporter dans l'espace, les différentes valeurs issues de la sommation des valeurs standardisées et pondérées de chaque critère intervenant dans l'élaboration de l'indicateur. Une reclassification des critères conduira à des cartes thématiques à quatre classes que sont les classes mauvaise ; moyenne ; bonne et excellente. Le nombre de classes est fixé à quatre pour une meilleure lisibilité et une bonne interprétation de la carte résultante (Youan Ta, 2008 ; Youan Ta et al., 2011). Toutes les combinaisons nécessaires pour l'établissement des cartes thématiques seront effectuées en mode " Raster » en utilisant l'outil « Map algebra » du module « Spatial Analyst » du logiciel Arcgis 10.2. Cette démarche conduira à la production des cartes thématiques DISPONIBILITE, EXPLOITABILITE, ACCESSIBILITÉ. Toutes ces cartes thématiques ont été combinées afin d'obtenir une carte des zones potentielles d'implantation de forages à gros débits.

\section{Validation de la carte des zones potentielles d'implantation de forages à gros débits}

Après l'établissement des différentes cartes thématiques, une validation de celles-ci s'impose afin d'apporter une crédibilité au travail effectué. Le critère d'évaluation choisi pour valider un indicateur donné doit être indépendant, c'est-à-dire n'ayant pas servi à la réalisation de cet indicateur et provenir exclusivement de la zone d'étude. La carte des zones potentielles d'implantation de forages à gros débits est validée en adoptant le mode de validation proposée par Dibi (2008). Ainsi, des débits de forages n'ayant pas servi à la réalisation de la carte d'exploitabilité ont été retenus puis superposés à la carte des zones potentielles d'implantation de forages à gros débits pour sa validation. Par ailleurs, pour respecter le principe de conformité du critère, des classes ayant les mêmes bornes que celles des cartes à évaluer sont définies. La carte des débits de forages regroupés en quatre classes (très fort, fort, moyen, faible) a donc été superposée à la carte des zones potentielles d'implantation de forages à gros débits. Il est relevé ensuite, pour chaque classe de sensibilité de la carte, le nombre de forages qui s'y trouvent selon leur classe. Le pourcentage de chaque classe de sensibilité et de facteur (débits) a été calculé par rapport au nombre total de forages obtenu par classe du facteur. Les courbes de tendances n'ont pas été utilisées compte tenu du nombre de données disponibles (Dibi, 2008).

\section{Résultats}

\section{Disponibilité des ressources en eaux souterraines}

La carte de disponibilité (figure 2) obtenue à partir de la combinaison des paramètres suivants : la densité de drainage, la densité de fracturation, l'épaisseur d'altération, l'infiltration efficace est dominée par la classe de 
disponibilité moyenne avec 79\% de couverture. Cette zone est caractérisée par une forte densité de drainage ce qui est défavorable à une alimentation de la nappe. Les classes de disponibilité bonne et excellente ne représentent que $3 \%$ de la zone d'étude. Elles traduisent une bonne alimentation des nappes; la fracturation y est intense et participe à la recharge de la nappe souterraine. Ces zones sont localisées au Sud autour de Dodougnoa, à l'Ouest et NordOuest de la zone d'étude.

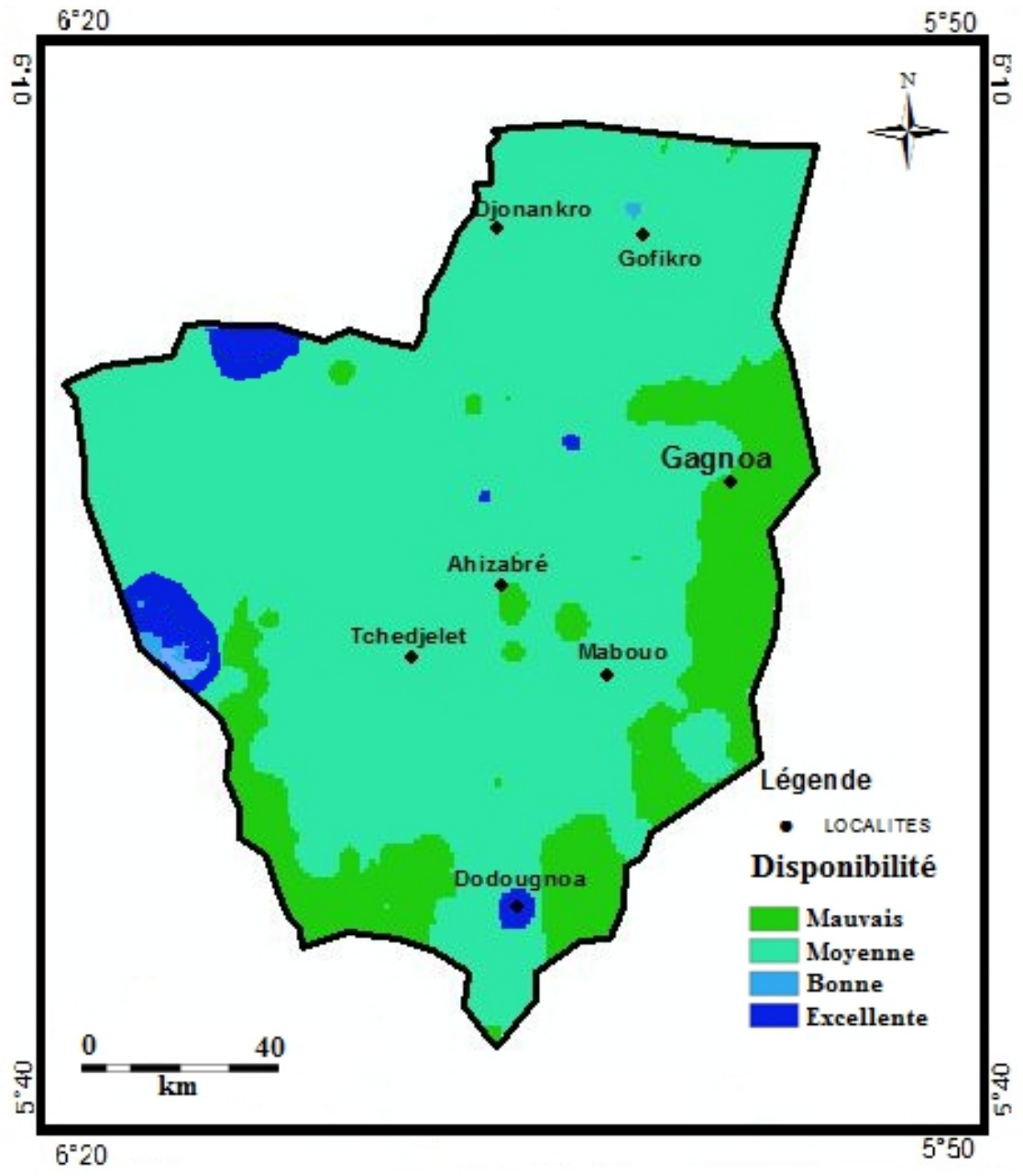

Figure 2 : Carte de disponibilité des ressources en eau de la région de Gagnoa

\section{Accessibilité aux ressources en eaux souterraines}

La combinaison des paramètres coût de foration et probabilité de succès a permis de générer la carte d'accessibilité de région de Gagnoa (Figure 3). Celle-ci est caractérisée par quatre classes d'inégale répartition : - Les zones d'accessibilité mauvaise représentent 7\% du domaine d'étude et correspondent aux zones à faible probabilité de succès (24\%) et où 
le coût de foration est supérieur à 1700000 F CFA (2 592 €). Ces zones se présentent en petits lambeaux disséminés sur la zone d'étude.

- $\quad$ Les zones d'accessibilité moyenne occupent 52\% de la zone d'étude et sont caractérisées par de faibles probabilités de succès (41\%) et un coût de foration relativement élevé autour de 1500000 (2 $287 €$ ). Elles sont localisées en grande partie au Nord, et à l'Ouest.

- $\quad$ Les zones d'accessibilité bonne couvrent 39\% de la zone d'étude et se situent au Sud-Ouest, au Nord-Est et dans la partie centrale. Dans ces zones, la probabilité de succès est élevée autour de $57 \%$ et la foration n'excède pas 1000000 F CFA (1 $524 €$ ).

- Les zones d'accessibilité excellente recouvrent seulement 2\% du territoire. Elles se présentent en petites plages localisées majoritairement au Sud-Est. Ces zones sont caractérisées par des coûts très faibles de foration (< 700000 F CFA (1 $067 €)$ ) et des probabilités très élevées de succès (>74\%).

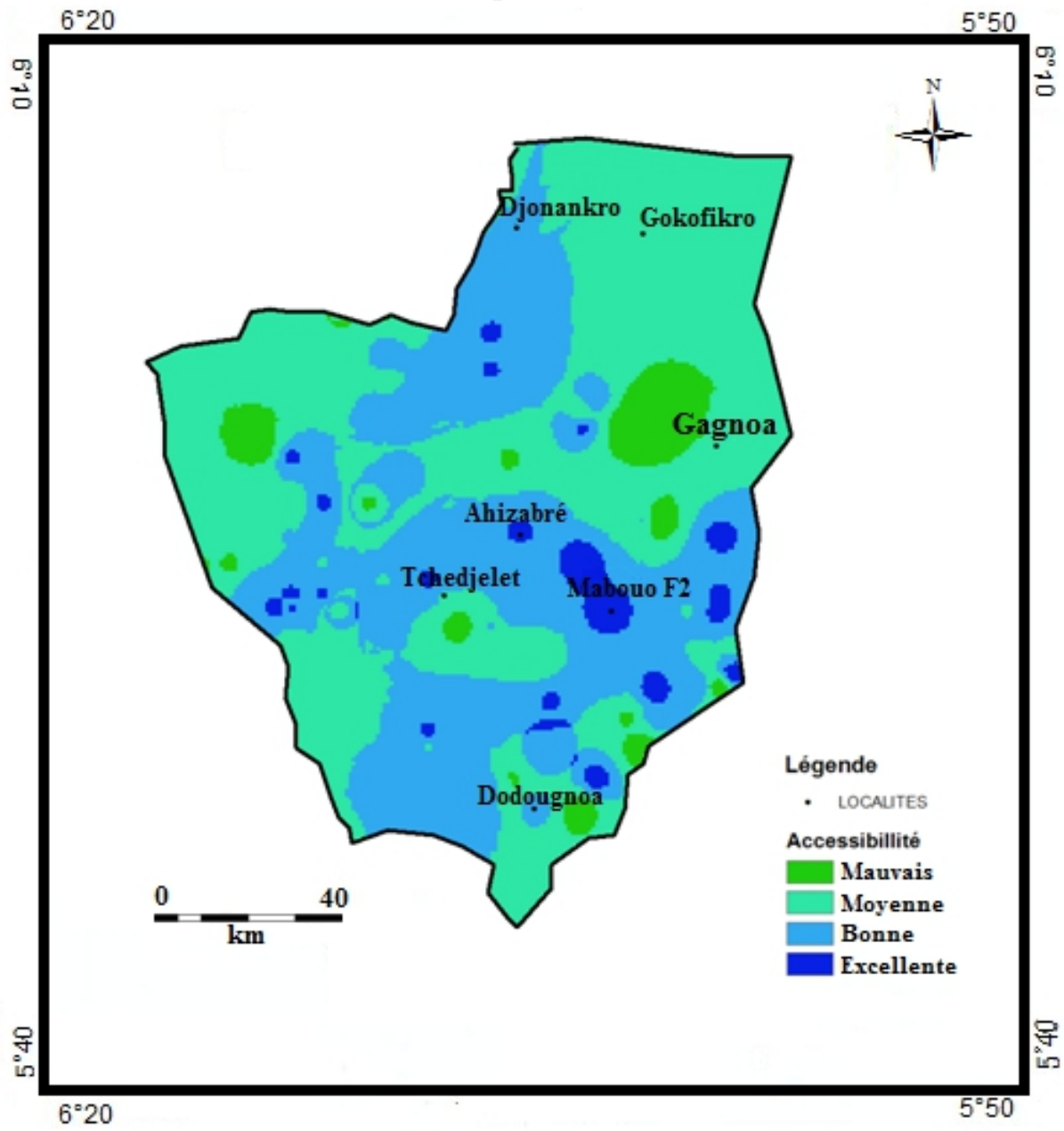

Figure 3 : Carte d’accessibilité des ressources en eau de la région de Gagnoa. 


\section{Exploitabilité des ressources en eaux souterraines}

La carte d'exploitabilité (figure 4) a été réalisée par l'agrégation appropriée du facteur débit et dans une moindre mesure des niveaux statistiques des ouvrages. L'analyse de cette carte thématique montre que les ressources en eaux souterraines de la zone d'étude sont difficilement exploitables. Les classes d'exploitabilité mauvaise et moyenne occupent une grande partie de la zone d'étude ( $90 \%$ de la zone d'étude). Les classes d'exploitabilité bonne et excellente sont représentées par une petite portion située au Sud de la zone d'étude autour de Dodougnoa.

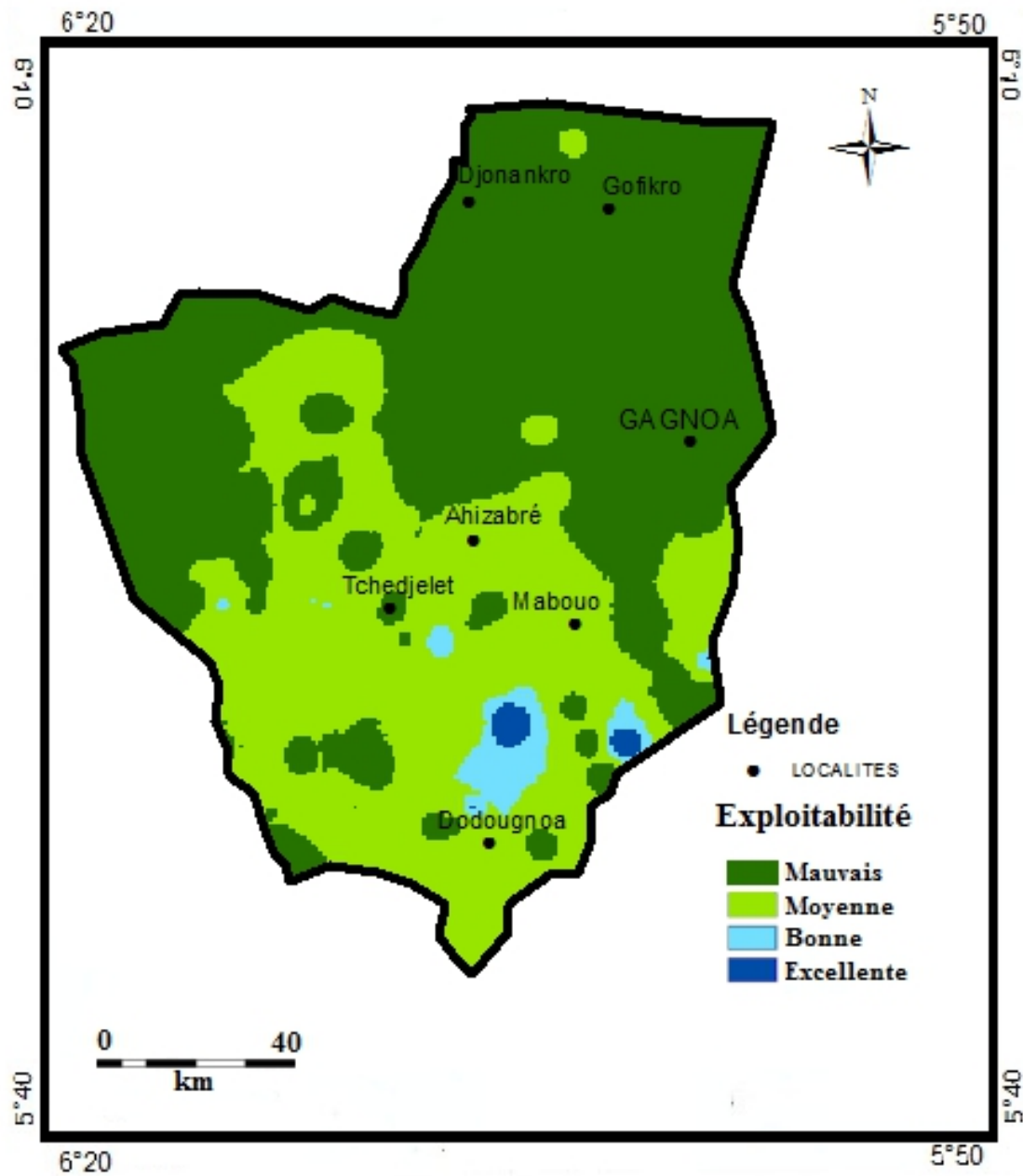

Figure 4 : Carte d'exploitabilité des ressources en eau de la région de Gagnoa.

\section{Zones potentielles d'implantation des points d'eau}

La combinaison des trois indicateurs que sont la disponibilité, l'exploitabilité et l'accessibilité a permis de produire la carte thématique des zones d'implantation (Figure 5). Les zones d'implantation mauvaises (35\%) et moyennes (41\%) sont majoritairement représentées sur l'ensemble de la 
zone d'étude (soit 76\%). Ce sont des zones à densité de fracturation faible et de profondeurs de forages élevées. Ces zones sont cependant appropriées pour l'implantation des forages dont les débits sont acceptables $\left(1 \mathrm{~m}^{3} / \mathrm{h}\right)$ dans le cadre de l'hydraulique villageoise.

Avec $24 \%$ de la superficie de la zone d'étude, les zones d'implantation bonne et excellente sont localisées au Centre et au Sud. Dans ces zones, les ouvrages sont moins profonds et avec une forte probabilité de succès. Ces zones sont caractérisées par une densité de fracturation élevée. La ressource en eau est disponible avec une pente relativement faible qui permet une bonne recharge de la nappe. Ces zones sont favorables à l'implantation de forages dans le cadre de l'hydraulique villageoise améliorée (HVA) ainsi que des forages à gros débits (hydraulique urbaine).

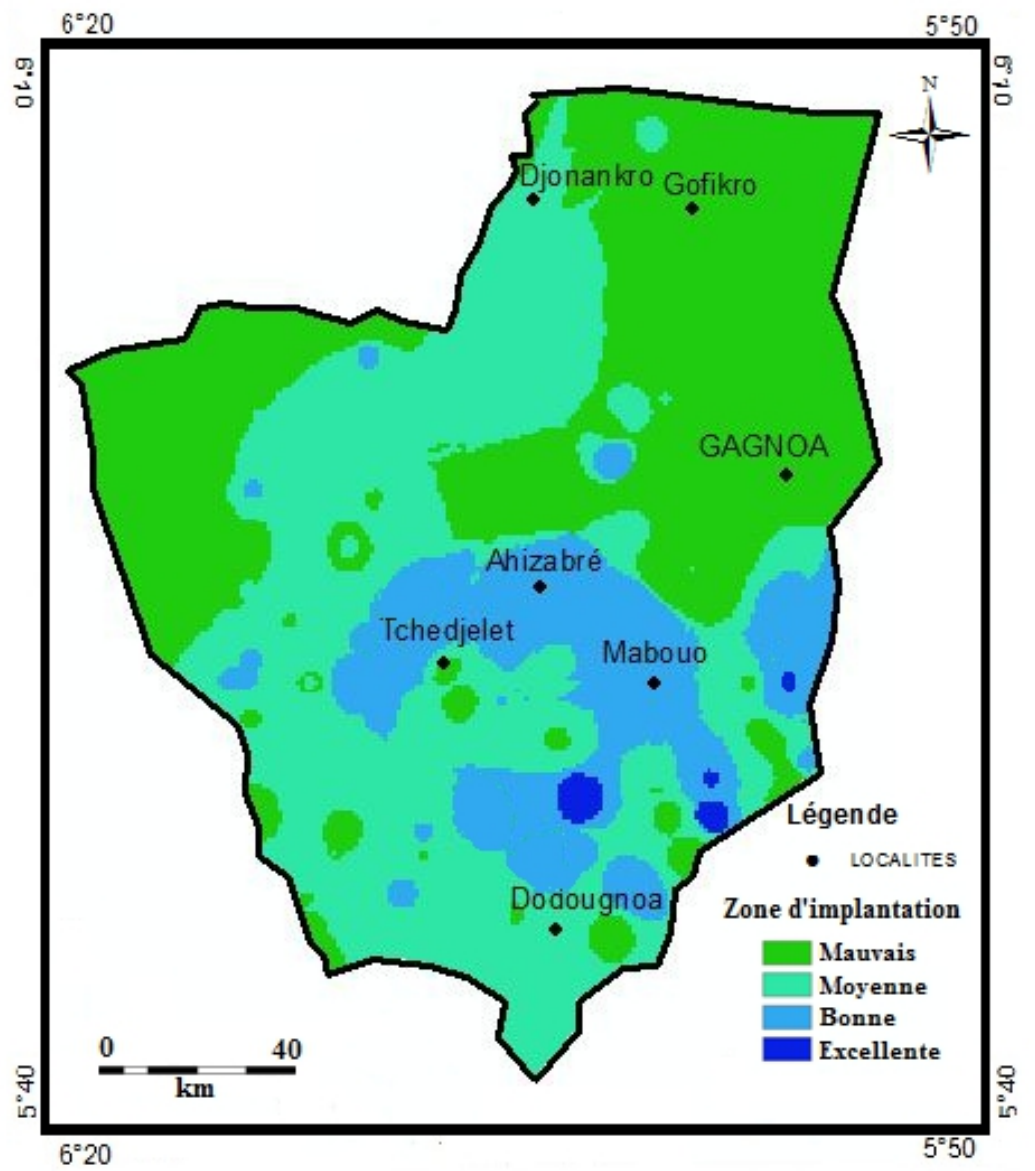

Figure 5 : Carte des zones d'implantation potentielles des forages à gros débits.

\section{Validation de la carte des zones potentielles d'implantation}

La validation de la carte des zones potentielles d'implantation s'est faite par sa superposition avec les forages réalisés par la société SOATH 
(Société Africaine de Travaux Hydrauliques) dans le cadre du projet CaféCacao pour la réalisation de forages d'hydraulique villageoise (figure 6). Le pourcentage de classe de sensibilité de débit se superposant à une classe thématique est représenté dans le tableau IV.

L'analyse de ce tableau montre que $16,67 \%$ des points d'eau à forts débits se superposent aux classes dites excellentes et $50 \%$ aux classes dites bonnes. Par ailleurs, il ressort que la totalité des forages (soit 100\%) avec un débit moyen coïncident avec la classe de sensibilité moyenne. Ceci vient renforcer l'intérêt qu'il faut porter à cette zone lors des campagnes d'hydraulique villageoise.

La classe de bonne sensibilité exprime mieux la potentialité des ressources en eaux souterraines bien que ces résultats soient nuancés par la représentativité de l'échantillon.

Au total, la plupart (66\%) des points d'eau, à débits supérieurs à 5 $\mathrm{m}^{3} / \mathrm{h}$, se superposent aux classes de sensibilité bonne et excellente.

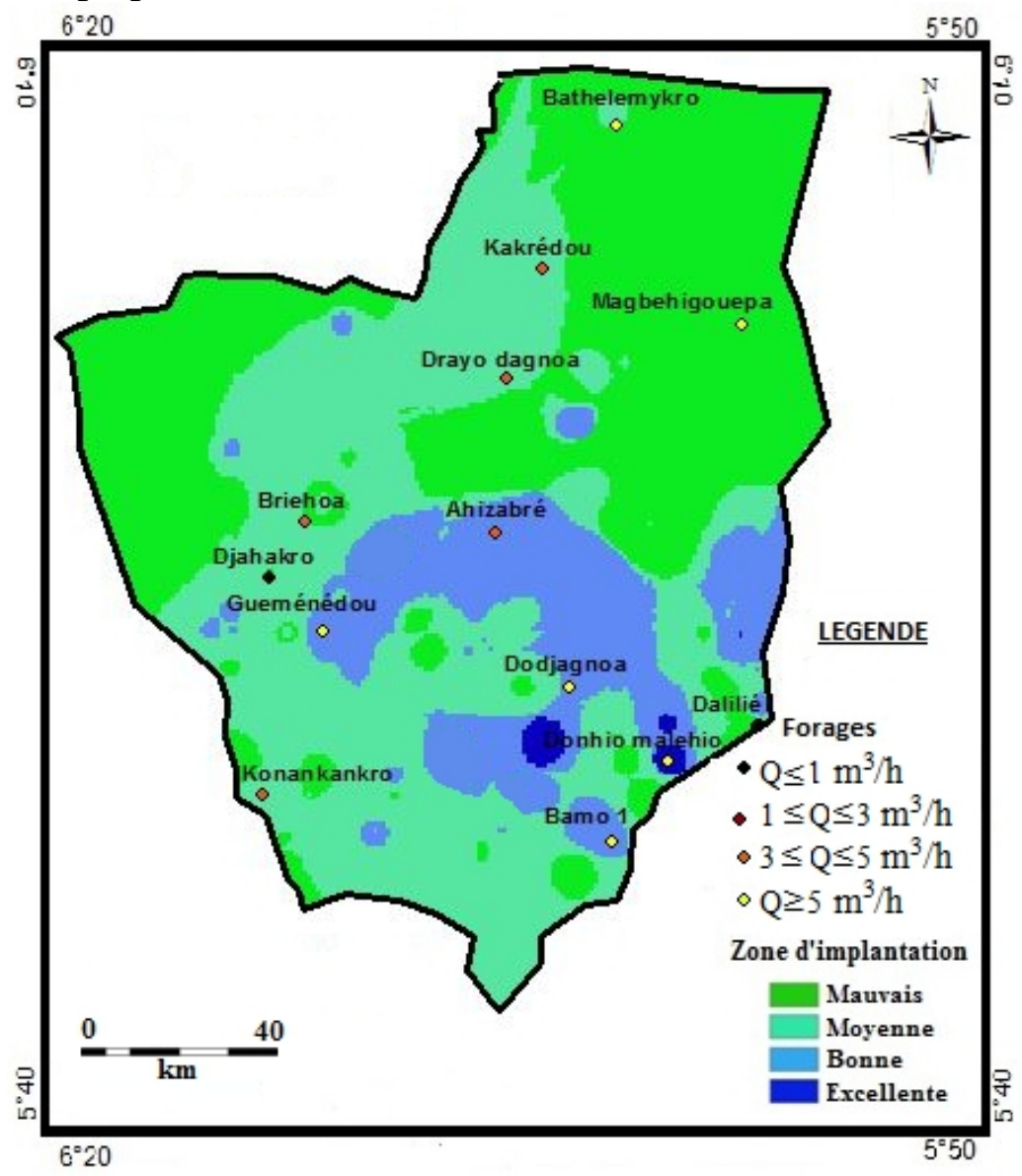

Figure 6 : Validation de la carte des zones d'implantation potentielle. 
Tableau IV: Pourcentage d'une classe de débit se superposant à une classe thématique

\begin{tabular}{ccccc}
\hline Classes de débits & $\begin{array}{c}\text { Très faible } \\
<1\end{array}$ & $\begin{array}{c}\text { Faible } \\
1-3\end{array}$ & $\begin{array}{c}\text { Moyen } \\
3-5\end{array}$ & $\begin{array}{c}\text { Fort } \\
>5\end{array}$ \\
Classes thématiques & & 0 & 0 & 16,66 \\
\hline Mauvaise & 33 & 100 & 100 & 16,66 \\
\hline Moyenne & 67 & 0 & 0 & 50,02 \\
\hline Bonne & 0 & 0 & 0 & 16,66 \\
\hline Excellente & 0 & & &
\end{tabular}

\section{Discussion}

Le SIG et la méthode d'analyse multicritère sont des outils de cartographie permettant la combinaison de plusieurs paramètres jugés aptes par son concepteur à rendre compte du phénomène étudié (Dibi, 2008). La validité du SIG est donc directement liée au choix de son initiateur pour la classification des différents paramètres utilisés (El Morjani, 2002 in Dibi, 2008). La méthode d'analyse multicritère ou plus exactement les méthodes d'aide multicritères à la décision ont été utilisées par de nombreux auteurs (Joerin, 1995, El Morjani, 2002, Jourda et al., 2006, Youan Ta et al., 2011). Elles ont permis la cartographie des zones favorables à l'implantation des forages à gros débits (Savané, 1997; Saley, 2003 ; Jourda, 2005) et la sélection de meilleurs sites de stockages de déchets (El Moorjani, 2003 ; Roy, 1997 ; Kouamé, 2007). Contrairement aux régions de Bondoukou (Youan Ta, 2008), Korhogo (Jourda, 2005) et Daoukro (Kanohin et al., 2012), la région de Gagnoa présente des zones d’implantation de forages à gros débits peu étendues dues essentiellement au caractère peu exploitable des ressources en eau de la zone. En plus de la fiabilité au niveau des données de débits, d’autres raisons pourraient éventuellement expliquer ce résultat. Il s’agit de :

- $\quad$ l'inégale répartition des données ayant servies à l'interpolation sur le secteur d'étude conduisant aux différentes cartes ;

- la considération de tous les critères comme parfaitement comparables. En effet, la pondération des critères qualitatifs est délicate contrairement aux critères quantitatifs (Joerin, 1995 in Saley, 2003). Toutefois, la technique de standardisation des critères facilite et rend possible leurs combinaisons ;

- $\quad$ le non-respect des limites des classes puisque ces limites de classes n’obéissent pas toujours à une classification universelle ;

- le manque de certaines données notamment les données hydroclimatiques et de mesures piézométriques en vue d’apprécier la recharge des nappes et de suivre leur évolution sur le terrain (Youan Ta, 2008).

Malgré toutes ces limites, le SIG constitue un apport dans la gestion des ressources en eau d'une manière générale. Il permet la manipulation d'un 
grand nombre de données de diverses natures et d'appréhender le problème posé dans tous ses aspects (Dibi, 2008). L'utilisation de plusieurs critères (9) regroupés en trois indicateurs (l'accessibilité, l'exploitabilité et la disponibilité) contribue à la fiabilité des résultats obtenus.

De ce fait, la carte des zones de potentialité en eau obtenue dans cette étude pourrait servir d'indicateur à toute prospection hydrogéologique comme le suggère Langevin et al. (1991). La validation de cette carte a montré que la carte obtenue dans la région de Gagnoa à l'instar des autres régions du pays reflète la réalité de terrain. Elle permettra de faciliter la prise de décision dans la recherche des eaux souterraines.

\section{Conclusion}

La synthèse des différentes cartes thématiques (Disponibilité, Accessibilité et Exploitabilité) a fait ressortir des zones de mauvaise, moyenne, bonne et d'excellente potentialité en eaux souterraines. Les zones favorables à l'implantation de forages à gros débits (classes de bonne et excellente) représentent $24 \%$ de la zone d'étude et sont localisées majoritairement dans la partie centrale. Cette étude constitue une contribution importante dans la connaissance des ressources en eau de cette région du pays.

\section{References:}

Akossi O. S., Lasm T., Youan Ta M., De Lasme O. Z., Kouamé F., Kouadio E., Baka D., Kouakou O. S., Yao F. A. (2014). Contribution of Remote Sensing and Geographic Information System to Identify Potential Areas of Groundwater in the Department of M'Bahiakro (Central-East of Côte d'Ivoire). British Journal of Applied Science \& Technology, Vol. 4, nº 18, pp. 2551-2575.

Dibi B. (2008). Cartographie des sites potentiels d'implantation des points d'eau dans le département d'Aboisso (Sud-Est de la Côte d'Ivoire) : apport du SIG et de l'analyse multicritère. Thèse de Doctorat, Université de Cocody, Abidjan, Côte d'Ivoire, 157p.

Eba L. (2013). Approche géomatique pour la délimitation des périmètres de protection autour des ressources en eau de surface destinées à la consommation: cas de la retenue d'eau de Gagnoa (Centre-Ouest de la Côte d'Ivoire), Thèse de Doctorat, Université Félix Houphouët-Boigny, Abidjan, Côte d'Ivoire, 180p.

El Morjani Z. (2003). Conception d'un système d'information à référence spatiale pour la gestion environnementale ; application à la sélection de sites potentiels de stockage de déchets ménagers et industriels en région semiaride (Souss, Maroc). Thèse de Doctorat, Université de Genève, 300p. 
Hentati I., Zairi M., Dhia H. B. (2005). Utilisation des SIG pour l'évaluation de la vulnérabilité environnementale des aquifères phréatiques : Cas de la nappe de Sfax- Agareb (Sud-Est tunisien. Communication SIG Environnement/Eau, Conférence Francophone ESRI, 2005.

INS (2015). Recensement général de la population et de l'habitat 2014. Région du Fromager, 40p.

Joerin F. (1995). Méthode multicritère d’aide à la décision et SIG pour la recherche d'un site. Revue internationale de géomatique, Vol. 5, $\mathrm{n}^{\circ}$. 1, pp. 37-51.

Jourda J. P., Saley M. B., Djagoua E. V., Kouame K. J., Biemi J., Razack M. (2006). Utilisation des données ETM+ de Landsat et d'un SIG pour l'évaluation du potentiel en eau souterraine dans le milieu fissuré précambrien de la région de Korhogo (Nord de la Côte d’Ivoire) : approche par analyse multicritère et test de validation. Télédétection, Vol. $5, n^{\circ} .4$, pp. 339-357.

Jourda J. P. (2005). Méthodologie d’application des techniques de télédétection et des systèmes d'information géographique à l'étude des aquifères fissurés d'Afrique de l'Ouest. Concept de l'hydrotechnique spatiale : cas des zones tests de la Côte d'Ivoire. Thèse de Doctorat d'Etat ès Sciences Naturelles, Université de Cocody-Abidjan, Côte d'Ivoire, 429p.

Kanohin F., Saley M., Aké E., Savané I. (2012). Apport de la télédétection et des SIG dans l'identification des ressources en eau souterraine dans la région de Daoukro (Centre-Est de la Côte d'Ivoire). International Journal of Innovation and Applied Studies, Vol. 1, pp. 35-53.

Kouamé K. J. (2007). Contribution à la gestion intégrée des ressources en eaux (GIRE) du District d'Abidjan (Sud de la Côte d'Ivoire): Outils d'aide à la décision pour la prévention et la protection des eaux souterraines contre la pollution. Thèse de Doctorat, Université de Cocody, Abidjan (Côte d'Ivoire), 222p.

Langevin C., Pernel F., Pointet T. (1991). Aide à la décision en matière de prospection hydrogéologique. L'analyse multicritère au service de l'évaluation du potentiel aquifère, en milieu fissuré (granite de Huelgoat, Finistère, France). Hydrogéologie, nº 1, pp. 51-64.

Roy B. (1995). Méthodologie multicritère d'aide à la décision. Economica, XXII, Paris, 423p.

Saaty T. L. (1977). A scaling method for priorities in hierarchical structures. Journal of Mathematical Psychology, n. ${ }^{\circ 15}$, pp. 234-281.

Saaty T. L. (2000). Fundamentals of Decision Making and Priority Theory with the Analytic Hierarchy Process, volume VI. Of AHP série

Saley M. B. (2003). Système d’informations hydrogéologiques à référence spatiale, discontinuités pseudo-images et cartographies thématiques des ressources en eau de la région semi- montagneuse de Man (Ouest de la Côte 
d'Ivoire). Thèse de Doctorat, Université de Cocody, Abidjan, Côte d'Ivoire, 211p.

Sorokoby V. M., Saley M. B., Kouame K. F., Djagoua M. E. V., Bernier M., Affian K., Biémi J. (2010). Utilisation des images Landsat ETM+ et du SHIRS pour la cartographie linéamentaire et thématique de Soubré-Meagui (Sud-Ouest de la Côte d'Ivoire): contribution à la gestion des ressources en eau souterraine. Télédétection, Vol. 9, nº. 3-4, pp. 209-223.

Wyns R., Gourry J. C, Baltassat J.-M., Lebert F. (1999). Caractérisation multi paramètres des horizons de subsufaces $(0-100 \mathrm{~m})$ en contexte de socle altéré. 2e colloque GEOFCAN BRGM IRD UMPC, Orléans, France, pp. 105-110.

Youan Ta M. (2008). Contribution de la télédétection et des systèmes d'informations géographiques à la prospection hydrogéologique du socle précambrien d'Afrique de l'Ouest: Cas de la région de Bondoukou Nord Est de la Côte d'Ivoire. Thèse de Doctorat, Université de Cocody, Abidjan (Côte d'Ivoire), 236p.

Youan Ta M., Lasm T., Jourda J P., Kouamé K. F., Razack M. (2008). Cartographie des accidents géologiques par imagerie Satellitaire landsat-7 $\mathrm{ETM}+$ et Analyse des réseaux de fractures Du socle précambrien de la région de Bondoukou (Nord-Est de la Côte d'Ivoire). Revue Télédétection, Vol. 8, $\mathrm{n}^{\circ}$. 2, pp. 119-135.

Youan Ta M., Lasm T., Jourda J. P., Saley M. B., Adja G. M., Kouamé K., Biémi J. (2011). Cartographie des eaux souterraines en milieu fissuré par analyse multicritère cas de Bondoukou (Côte d'Ivoire). Revue Internationale de Géomatique. Vol. 21, nº 1, pp. 43-71.

Youan Ta M., De Lasme O. Z., Baka D., Lasm T., Jourda J. P., Biémi J. (2015). Analyse des propriétés hydrodynamiques de l'aquifère fissuré du socle Paléo protérozoïque : Aide à l'approvisionnement en eau potable des populations de la région de Bondoukou (Nord-Est de la Côte d'Ivoire). International Journal of Innovation and Applied Studies Vol. 13, pp. 561580 . 